Original

\title{
Análisis de las aguas embotelladas y de grifo españolas y de las implicaciones de su consumo en la litiasis urinaria
}

\author{
Félix Millán Rodríguez, Silvia Gracia García*, Rocío Jiménez Corro, Miriam Serrano Liesa, \\ Ferran Rousaud Barón, Francisco Sánchez Martín, Oriol Angerri Feu, Roberto Martínez Rodríguez, \\ Humberto Villavicencio Mavrich
}

Servicio de Urología. *Servicio de Bioquímica. Fundació Puigvert. Barcelona, España.

\section{Resumen}

Objetivo: Realizar un análisis de la composición de las aguas disponibles en España para ofrecer unas recomendaciones de consumo a los pacientes litiásicos.

Material y Métodos: Búsqueda de información sobre la composición de aguas de grifo y embotelladas en publicaciones, supermercados e Internet. Estudio descriptivo y de la correlación entre los distintos componentes mediante el coeficiente de correlación de Pearson.

Resultados: Se obtuvo información sobre la composición de agua de grifo de la mayoría de capitales españolas y de 85 marcas de agua embotellada. Se encontró una correlación entre la concentración de calcio y magnesio ( $\mathrm{p}=0,0001)$ y una intensa relación entre la de bicarbonato y sodio ( $\mathrm{p}=0,0001$, coeficiente Pearson 0,958$)$. Se ofrece clasificación de las aguas en función de la concentración de calcio, bicarbonato, sodio y magnesio.

Conclusión: Se propone una guía de elección de agua del paciente litiásico en función de su procedencia geográfica y de sus preferencias dietéticas, principalmente el consumo de lácteos.

Palabras clave: Agua embotellada. Agua de grifo. Litiasis urinaria. Calcio. Bicarbonato. Sodio. Recomendaciones dietéticas.

\section{Spanish bottled and tap water analysis and their relation with urinary lithiasis}

\section{Abstract}

Objective: To perform a chemical analysis of all the available waters in Spain with the idea of offering consume recommendations to lithiasic patients.

Material and Methods: Information research of the chemical composition of Spanish tap and bottled water in publications, supermarkets and Internet. A descriptive study, and a correlation study between water components by means of Pearson test were performed.

Results: Information about composition from tap water of most of the main Spanish cities and 85 bottled water brands was found. A significant correlation between calcium and magnesium concentration $(\mathrm{p}=0.0001)$ and high correlation between bicarbonate and sodium concentration ( $\mathrm{p}=0.0001$, Pearson coefficient 0.958 ) was found. It is also offered water classifications according to calcium, bicarbonate, sodium and magnesium concentrations.

Conclusion: A guideline about water election for lithiasic patients is offered according to their geographical origin and dietetic preferences, mainly lactic consume.

Keywords: Bottled water. Tap water. Urinary lithiasis. Calcium. Bicarbonate. Sodium. Dietetic recommendations.

\section{INTRODUCCIÓN \\ Agua y litiasis renal}

El agua representa el 60\% de todo el peso corporal, siendo un $26 \%$ extracelular y un 34\% intracelular. Así, una persona de $75 \mathrm{~kg}$ de peso, tendría 451 de agua, y aproximadamente unas pérdidas diarias de $2.500 \mathrm{ml}$ ( $1.500 \mathrm{ml}$ orina, $900 \mathrm{ml}$ pérdidas insensibles y sudor, $100 \mathrm{ml}$ heces). Por otro lado, el metabolismo proporciona una producción endógena de $350 \mathrm{ml}$ diarios de agua, por lo que se necesitaría una aportación exógena de $2.150 \mathrm{ml}$ para cubrir las pérdidas. Si la comida (principalmente frutas $\mathrm{y}$ vegetales) aportan 500-900 $\mathrm{ml}$ diarios de agua, esto significa que para cubrir las pérdidas hemos de ingerir diariamente un mínimo de 1.250-1.650 ml de agua ${ }^{1}$. Obviamente, estos valores dependerán de la edad, el peso, la actividad física y las condiciones climatológicas de cada persona, aunque hay expertos en nutrición que recomiendan una ingesta de agua de unos $30 \mathrm{ml} / \mathrm{Kg} /$ día en adultos ${ }^{2}$. Siguiendo 
con este ejemplo, se observa que los 850-1.250 ml que aportan diariamente la ingesta de alimentos y la producción endógena de agua pueden ser suficientes para compensar los $1.000 \mathrm{ml}$ de pérdidas insensibles y por heces, por lo que la mayor parte del aporte que se realice de agua va a ir a la formación de orina. De esta forma, una persona con una baja ingesta de agua no va a poner en peligro el equilibrio hidroelectrolítico, pero sí que producirá una orina sobresaturada teniendo un riesgo mayor de formación de litiasis.

Nuestra experiencia clínica diaria nos muestra que un alto porcentaje de los pacientes litiásicos a los que se analiza la orina de 24 horas tienen unos volúmenes de diuresis bajos. En este sentido, hay estudios que demuestran que los pacientes con litiasis cálcica idiopática tienen una diuresis media ( $1.000 \mathrm{ml}$ ) inferior a la del grupo control de pacientes no litiásicos $(1.400 \mathrm{ml})^{3}$. Este mismo estudio, evidenció también que en los pacientes con litiasis cálcicas idiopáticas, el aumento de la ingesta líquida hasta obtener diuresis de $2.000 \mathrm{ml}$, sin necesidad de realizar otros cambios dietéticos, consiguió una disminución y un retraso significativo de la recurrencia litiásica. Un estudio de Curhan et $\mathrm{al}^{4}$ en varones sin antecedentes litiásicos, demostró que una ingesta media de agua de 2.000-2.500 ml y una ingesta superior a $2.500 \mathrm{ml}$ disminuían respectivamente la incidencia de litiasis un $11 \%$ y un $29 \%$ en comparación a los que tomaban menos de $1.300 \mathrm{ml}$ diarios. Por otro lado, tal como muestran los trabajos de García Matilla ${ }^{5,6}$ y de Medina López ${ }^{7}$, otro de los beneficios del aumento de la ingesta líquida es el incremento del $\mathrm{pH}$ urinario.

Aunque el aumento de la ingesta líquida con el fin de obtener un aumento de la diuresis, es la primera medida que un urólogo recomienda a todo paciente litiásico para disminuir la recurrencia, con frecuencia, aparecen ciertas dudas a la hora de recomendar un tipo concreto de agua, que se incrementan ante la gran variedad y diferencias de aguas del grifo y embotelladas que existen en nuestro país. El objetivo de este trabajo es, precisamente, realizar un análisis de la composición de todas las aguas disponibles en España, y ofrecer una guía de recomendaciones individualizada en función del tipo de litiasis, los hábitos dietéticos, la procedencia geográfica y los hábitos de consumo de cada paciente. Con esto conseguiremos, desde las Unidades de Litiasis, realizar recomendaciones dietéticas perso- nalizadas que aporten a los pacientes un mayor beneficio que el clásico "Beba usted mucha agua".

Sin embargo, dado que el efecto de la ingesta de agua sobre la litiasis renal no depende sólo del volumen ingerido sino de la concentración de calcio, bicarbonato y sodio, a continuación se va a analizar el efecto de estos iones en la formación de la litiasis, para así, más adelante, disponer de los elementos de juicio necesarios para recomendar un tipo de agua en concreto.

\section{Calcio y litiasis renal}

La dosis diaria recomendada de ingesta de calcio es de 1.000 mg. Dado que muchos casos de litiasis de oxalato cálcico se asocian a hipercalciuria, hace años se pensaba que una dieta de bajo contenido en calcio, disminuiría la hipercalciuria y por tanto, la recurrencia de litiasis oxalocálcica. Sin embargo, varios estudios demostraron posteriormente que dicha teoría no sólo era falsa, sino que se producía el efecto contrario ${ }^{4,8,9}$. Así, Curhan et $\mathrm{al}^{4}$ lo comprobaron en una cohorte de varones mayores de 40 años sin antecedentes litiásicos, donde el riesgo de formación de litiasis disminuía a medida que aumentaba la ingesta de calcio en la dieta, siendo un $32 \%$ y un $34 \%$ menor entre los que tomaban 700-1.000 mg o más de 1.000 mg diarios de calcio, respectivamente, en comparación a los que tomaban menos de $600 \mathrm{mg}$ diarios de calcio. Años más tarde, Borghi et $\mathrm{al}^{8}$ observaron que los varones con hipercalciuria formadores de litiasis oxalocálcica recurrente que seguian una dieta baja en proteínas animales y sal pero con toma normal de calcio, presentaban una menor recurrencia que aquellos que seguían una dieta baja en calcio. Un resultado similar se encontró en otro estudio realizado entre mujeres $^{9}$. La explicación fisiopatológica de este hecho se basa en que dado que el oxalato se une al calcio a nivel intestinal, una dieta baja en calcio conlleva el disponer de más oxalato libre en el intestino. De este modo, aumenta la absorción intestinal de oxalato y por tanto la oxaluria y debido a que en la formación de la litiasis oxalocálcica la oxaluria es más determinante que la calciuria $^{10}$, al final se ve aumentado el riesgo de formación de litiasis oxalocálcica. No obstante, cabe cuestionarse en el trabajo de Borghi et $\mathrm{al}^{8}$, si la disminución de la recurrencia se debe al hecho de tomar una dieta normal en calcio o también al hecho de hacer una dieta baja en proteínas animales y sal. 
La relación de la concentración de calcio en el agua con ciertas enfermedades ha sido estudiada principalmente en el campo de la litiasis renal y en el de la osteoporosis ${ }^{11,12}$. Así, mientras es importante asegurar una ingesta adecuada de calcio en las pacientes con osteoporosis ${ }^{12}$ también ha de tenerse en cuenta que para favorecer su absorción, los bifosfonatos se han de tomar con aguas bajas en calcio porque sino forman sales insolubles ${ }^{11}$.

En cuanto al tema que nos ocupa, hay varios trabajos que comparan la influencia de determinados tipos de agua en la formación de litiasis ${ }^{13-16}$. Caudarella et al $^{13}$ compararon 3 tipos de agua y observaron que la que tenía un mayor contenido de calcio consiguió una disminución de la oxaluria. No obstante, hay que tener en cuenta que esta agua presentaba un mayor contenido de bicarbonato, que produjo un aumento significativo de la citraturia, que puede por sí solo contribuir a la disminución de la incidencia de litiasis. Por ello, a la hora de analizar un tipo de agua en concreto no ha de tenerse en cuenta sólo la concentración de calcio sino la de otros iones como el bicarbonato, el sodio o el magnesio que también pueden influir en la formación de litiasis, como veremos a continuación.

\section{Bicarbonato y litiasis renal}

La ingesta de bicarbonato y por tanto de la de aguas ricas en bicarbonato, debido a su acción alcalinizante constituye un recurso terapéutico eficaz en el tratamiento de la litiasis úrica, consiguiéndose en ocasiones, la disolución de la litiasis. No obstante, el principal problema de las aguas con alto contenido en bicarbonato, es su alto contenido en sodio por lo que no es aconsejable un uso prolongado en pacientes litiásicos, y más aún si son hipertensos.

Por otro lado, existen también estudios que han valorado la acción de las aguas bicarbonatadas sobre la litiasis de oxalato cálcico ${ }^{15-17}$, pero desafortunadamente, basados en pocos casos. El trabajo de Coen et $\mathrm{al}^{15}$, muestra un efecto beneficioso sobre los parámetros urinarios en un pequeño grupo de 11 voluntarios sanos que tomó agua con una gran concentración de bicarbonato, de calcio y baja en sodio. Karagülle et $\mathrm{al}^{16}$ realizaron un estudio en 34 pacientes de alto riesgo litiásico administrando un agua con alto contenido en bicarbonato y magnesio, observando una disminución de la concentración de oxalato cálcico que también se dio en el grupo control. El interés del trabajo de Siener et al ${ }^{17}$ en el que se evalúa el efecto de la ingesta de agua rica en bicarbonato y magnesio en 12 voluntarios sanos, es la descripción de los ritmos circadianos de los diferentes solutos urinarios.

En resumen, aunque la toma de bicarbonato provoque un aumento de la citraturia que beneficie a los pacientes afectos de litiasis oxalocálcica, el efecto terapéutico más importante lo vamos a encontrar en el tratamiento de la litiasis úrica debido a su papel alcalinizante.

\section{Sodio y litiasis renal}

Existen varios trabajos que muestran la relación entre la ingesta de sodio y la excreción renal de calcio $^{18-21}$, por lo que en los pacientes litiásicos hay que recomendar dietas bajas en sal, y en consecuencia, aguas con un bajo contenido en sodio. La ingesta de sodio diaria debería oscilar entre los 500 y los $3.000 \mathrm{mg}^{2}$. Sin embargo, según la guía del "Tratamiento no farmacológico de la hipertensión arterial" de la Sociedad Española de Hipertensión ${ }^{22}$ los pacientes hipertensos deberían consumir menos de $5 \mathrm{~g}$ diarios de $\mathrm{NaCl}$, lo que equivale a menos de 2 $\mathrm{g}$ de sodio diario.

\section{MATERIAL Y MÉTODOS}

Se ha realizado una búsqueda lo más exhaustiva posible de las aguas de grifo y embotelladas que se producen en España. En cuanto a las aguas embotelladas, el criterio de inclusión ha sido que sean obtenidas en manantiales de nuestro país, por ello se han excluido del estudio todas aquellas aguas extranjeras comercializadas en nuestro país (ejemplo Evian, Perrier...). El motivo es que si se hubiesen incluido las aguas extranjeras, la lista de posibles marcas de agua podría ser desmesurada para nuestro análisis.

La información sobre el análisis del agua de grifo se ha buscado de forma secundaria en trabajos previos publicados que ya habian realizado esta investigación. La información sobre el análisis de las aguas embotelladas se ha realizado a partir de la información de la etiqueta de las botellas, tras visitar los principales supermercados e hipermercados existentes en nuestra ciudad, Barcelona. La búsqueda se amplió también, con la información obtenida en trabajos previos publicados con objetivos parecidos, en las páginas propias de las marcas buscadas por Internet y en páginas de Internet como www.aquamania.net. En caso de discrepancia en una marca concreta, entre la información aportada 
por la etiqueta y la obtenida por Internet o secundariamente de una publicación previa, se usó la información de la etiqueta por entender que es la más actualizada. Se creo una base de datos con todas las marcas de agua embotellada donde se recogieron todos los parámetros del análisis químico, aunque para nuestro estudio se analizó las concentraciones de calcio, sodio, bicarbonato y magnesio.

Finalmente, se solicitó información a las empresas Grupo Corsa (tratamiento del agua de grifo mediante osmosis inversa) y Brita (filtros para el agua del grifo) sobre trabajos que muestren qué cambios químicos en el agua de grifo comportan el uso de sus productos.

En cuanto al estudio estadístico, se realizó en primer lugar, un estudio descriptivo de las concentraciones de iones en cada tipo de agua y en segundo lugar, un estudio de correlación mediante el coeficiente de Pearson, para ver si había una asociación entre las distintas concentraciones de iones.

\section{RESULTADOS}

Se recogió información sobre 85 marcas de agua embotellada, encontrándose en la mayoría de casos información sobre la concentración de calcio, bicarbonato, sodio, magnesio, sulfato y cloruros, mientras que en muchas de ellas faltaba información sobre el residuo seco, el sílice y el potasio (Tabla 1).

Tabla 1. Análisis Químico de 85 Aguas Embotelladas, Estudio Descriptivo (mg/l)

\begin{tabular}{|c|c|c|c|c|}
\hline Elemento & $\begin{array}{l}\text { Media } \\
\left(\mathrm{IC}_{95 \%}\right)\end{array}$ & Mínimo & Máximo & $\begin{array}{c}\mathbf{N}^{\circ} \text { casos } \\
\text { desconocido }\end{array}$ \\
\hline Calcio & $\begin{array}{c}57,9 \\
(49,5-66,2)\end{array}$ & 0,5 & 181 & 1 \\
\hline Bicarbonato & $\begin{array}{c}295,2 \\
(208,3-382,1)\end{array}$ & 2,1 & 2.196 & 1 \\
\hline Sodio & $\begin{array}{c}67,3 \\
(19,9-114,8)\end{array}$ & 0,6 & 1.136 & 4 \\
\hline Magnesio & $\begin{array}{c}18,0 \\
(14,3-21,7)\end{array}$ & 0,3 & 65,6 & 5 \\
\hline Residuo seco & $\begin{array}{c}343,5 \\
(225,0-461,9)\end{array}$ & 25,0 & 3.094 & 27 \\
\hline Sulfatos & $\begin{array}{c}49,4 \\
(31,1-67,8)\end{array}$ & 1,2 & 459 & 8 \\
\hline Cloruros & $\begin{array}{c}49,6 \\
(22,4-76,9)\end{array}$ & 0,8 & 610 & 9 \\
\hline Sílice & $\begin{array}{c}18,6 \\
(12,6-24,7)\end{array}$ & 1,3 & 113 & 38 \\
\hline Potasio & $\begin{array}{c}4,7 \\
(0,7-8,7)\end{array}$ & 0,2 & 50,7 & 51 \\
\hline
\end{tabular}

Se presentan las aguas ordenadas en sentido descendente en cuanto a su concentración en $\mathrm{mg} / \mathrm{l} \mathrm{de}$ calcio (Tabla 2), bicarbonato (Tabla 3), sodio (Tabla 4) y magnesio (Tabla 5).

Cuando se analizó la correlación entre los principales elementos químicos de las aguas embotelladas (Tabla 6), se encontró una correlación estadísticamente significativa entre el contenido en calcio y magnesio ( $\mathrm{p}=0,0001)$ y entre el contenido en bicarbonato y sodio $(\mathrm{p}=0,0001)$, esta última con un altísimo coeficiente de correlación de Pearson $(0,958)$.

Para conocer las características del agua de grifo de las distintas capitales de nuestro país se recurrió a fuentes secundarias, encontrando dos trabajos. El primero de Martínez-Ferrer et al $^{12}$ centrado en la osteoporosis y el segundo de Vitoria y $\operatorname{Arias}^{23}$ en la nutrición infantil (Tabla 7). Aunque existen pocas discrepancias entre los datos de ambos trabajos, el de MartínezFerrer es más actual y dispone de la información de casi todas las capitales españolas, mientras que el de Vitoria y Arias no tiene información de todas las capitales pero sí que aporta información sobre un gran número de municipios españoles, incluso algunos menores de 1.000 habitantes.

Finalmente, las empresas Grupo Corsa y Brita no ofrecieron información sobre sus productos a pesar de haberla solicitado.

\section{DISCUSIÓN}

Las aguas comercializadas en España provienen principalmente de manantiales, de los que disponemos en gran número y variedad en nuestro país, por lo que estas aguas son usadas no sólo para el consumo sino también con fines termales. Debido a ello, se dispone de estudios geológicos como el de Martín-Gil et $\mathrm{al}^{24}$ que extrae unas conclusiones muy interesantes sobre las aguas de nuestro país:

- Las aguas menos mineralizadas son las de la Sierra de Guadarrama y las más mineralizadas las del Sistema Ibérico.

- Si se comparan aguas de localizaciones próximas, las situadas al Sur tienen más contenido de $\mathrm{NaCl}$ que las situadas al Norte, cuanto más al oeste más mineralizadas, y cuanto más profundas mayor contenido en bicarbonatos. 
Tabla 2. Aguas embotelladas ordenadas en sentido descendente según el contenido en Calcio (mg/l)

\begin{tabular}{|c|c|c|c|c|}
\hline Marca & $\mathbf{C a}$ & $\mathbf{N a}$ & Bicarbonato & Mg \\
\hline San Pellegrino & 181,0 & 36,1 & 239,0 & 53,5 \\
\hline Insalus & 157,3 & 10,0 & 156,4 & 19,3 \\
\hline $\mathrm{Na}$ Taconera & 144,0 & 49,0 & 275,0 & 28,0 \\
\hline Agua de Serria Balnéa & 118,0 & - & 239,0 & 51,0 \\
\hline Agua de Sierra & 118,0 & - & 239,0 & 51,0 \\
\hline Font Teca & 115,0 & - & 244,0 & 51,0 \\
\hline Lunares & 102,7 & 39,5 & 296,3 & 36,7 \\
\hline Font Jaraba & 100,3 & 42,5 & 301,3 & 40,9 \\
\hline $\mathrm{El}$ portell & 95,4 & 23,6 & 257,4 & 12,2 \\
\hline Vitel & 94,0 & 7,7 & 248,0 & 20,0 \\
\hline Monte Pinos & 93,8 & 1,8 & 298,0 & 3,4 \\
\hline Monte Pinos & 93,8 & 1,8 & 298,0 & 3,4 \\
\hline Fontecabras & 93,0 &, 0 & 300,7 & 38,9 \\
\hline Benassal & 92,2 & 2,7 & 267,2 & - \\
\hline Sant Aniol & 92,1 & 6,0 & 343,1 & 15,4 \\
\hline Aigua mineral Bonpreu & 92,1 & 6,3 & 345,3 & 17,0 \\
\hline Aquabona & 90,3 & 2,4 & 277,0 & 2,8 \\
\hline Santolin & 89,8 & 2,6 & 266,0 & 2,4 \\
\hline Font del Cami & 87,4 & 39,3 & 379,6 & 43,3 \\
\hline Fuente Primavera & 86,6 & 20,7 & 295,3 & 23,3 \\
\hline Agua del Rosal & 85,7 & 7,2 & 400,2 & 33,5 \\
\hline Agua del Valle del Cardo & 85,7 & 7,2 & 400,2 & 33,5 \\
\hline Vilajuiga & 83,4 & 568,0 & $1.561,6$ & 46,7 \\
\hline Cortés & 81,9 & 6,4 & 268,4 & 7,2 \\
\hline Fonteforte & 80,6 & 108,5 & 205,6 & 19,9 \\
\hline Aquadeus & 80,2 & 3,7 & 345,5 & 30,5 \\
\hline Firgas & 79,7 & 62,9 & 513,8 & 41,4 \\
\hline Sierra de Cazorla & 77,8 & 1,3 & 465,4 & 37,1 \\
\hline Castro Vita & 76,5 & 8,6 & 240,3 & 16,1 \\
\hline Pineo & 74,0 & 1,5 & 238,0 & 4,1 \\
\hline Font De Pla Nova & 74,0 & 11,0 & 270,0 & 36,0 \\
\hline Solares & 73,7 & 87,6 & 248,9 & 15,6 \\
\hline Fuensanta & 71,3 & 15,9 & 222,2 & 10,9 \\
\hline Marquesado & 70,5 & ,8 & 267,7 & 18,5 \\
\hline Ribagorza & 69,8 & 25,3 & 331,8 & 25,2 \\
\hline Veri & 69,0 & ,6 & 197,0 & 1,5 \\
\hline Fuente Solana & 67,1 & 19,2 & 226,3 & 18,0 \\
\hline Font del Pi & 66,5 & 26,9 & 316,6 & 65,6 \\
\hline Agua del Rosal & 66,2 & 49,8 & 280,3 & 11,1 \\
\hline Fuente Liviana & 65,4 &, 8 & 268,3 & 17,0 \\
\hline Agua mineral Carrefour & 64,1 &, 8 & 225,1 & 18,5 \\
\hline Pallars & 62,5 & 67,0 & 140,3 & 22,4 \\
\hline Fuentedueñas & 59,6 & 5,6 & 226,0 & 8,4 \\
\hline Solan de Cabras & 56,9 & 5,3 & 285,0 & 25,5 \\
\hline Agua de Ribes & 54,4 & 4,9 & 163,5 & 7,5 \\
\hline Font Agudes del Montseny & 53,8 & 41,3 & 252,2 & 14,3 \\
\hline
\end{tabular}


Tabla 2. (Continuación)

\begin{tabular}{|c|c|c|c|c|}
\hline Marca & $\mathbf{C a}$ & $\mathbf{N a}$ & Bicarbonato & Mg \\
\hline San Narciso & 51,4 & $1.080,0$ & $2.196,0$ & - \\
\hline Fondalt & 50,5 & 5,0 & 163,6 & 15,8 \\
\hline Malavella & 48,9 & $1.136,0$ & $2.147,0$ & 8,1 \\
\hline Agua de Cuevas & 47,0 & 1,6 & 246,9 & 25,4 \\
\hline Agua Alzola & 44,0 & 44,0 & 192,0 & 5,8 \\
\hline Agua San Joaquín & 41,2 & 10,4 & 152,3 & 12,3 \\
\hline Font Vella & 38,5 & 13,2 & 149,0 & 9,7 \\
\hline Fontanel & 36,3 & 39,6 &, 0 & 10,9 \\
\hline Font del Regàs & 36,2 & 14,7 & 133,1 & 3,4 \\
\hline Sierres de Jaen & 36,0 & 1,8 & 135,0 & 15,0 \\
\hline Aquarel & 34,0 & 10,0 & 115,0 & 4,0 \\
\hline Agua mineral Auchan & 33,9 & 49,7 & 213,8 & 5,0 \\
\hline Fonter & 32,0 & 7,4 & 127,0 & 7,3 \\
\hline Azuébar & 28,5 & 7,7 & 200,0 & - \\
\hline Les Creus & 28,0 & 11,7 & 119,0 & 7,3 \\
\hline Lanjarón & 27,2 & 4,8 & 105,0 & 8,8 \\
\hline Viladrau & 27,0 & 9,6 & 112,3 & 4,8 \\
\hline Carrizal & 27,0 & 1,0 & 104,0 & 6,0 \\
\hline Fontecelta & 26,5 & 89,5 & 288,3 & - \\
\hline Font d`Or & 26,4 & 9,2 & 78,8 & 3,2 \\
\hline Valtorre & 25,6 & 30,5 & 191,0 & 23,6 \\
\hline San Vicente & 22,0 & 5,9 & 81,1 & 7,3 \\
\hline La Platina & 16,0 & 9,4 & 78,7 & 15,8 \\
\hline Aigua del Montseny & 13,1 & 9,8 & 48,7 & 4,2 \\
\hline San Carlos & 12,0 & 31,0 & 21,0 & 4,3 \\
\hline Agua de Borines & 9,6 & 35,3 & 105,8 & 2,9 \\
\hline Cabreiroá & 9,5 & 49,3 & 162,3 & 4,5 \\
\hline Mondariz & 9,2 & 50,5 & 163,4 & 4,9 \\
\hline Caldes de Boí & 7,3 & 39,2 & 38,7 &, 7 \\
\hline Fonteide & 6,4 & 19,1 & 57,1 & 3,7 \\
\hline Font Selva & 6,0 & 1,0 & 249,0 & 54,9 \\
\hline Fonsana & 5,3 & 7,0 & 38,0 &, 9 \\
\hline Calabor & 5,1 & 47,7 & 115,0 & ,9 \\
\hline Sousas & 3,4 & 29,0 & 79,8 &, 9 \\
\hline Aguavida & 2,8 & 7,2 & 171,0 & 57,1 \\
\hline Bezoya & 1,6 & 2,3 & 6,7 & ,3 \\
\hline Agua sana &, 5 & 5,9 & 4,6 & ,8 \\
\hline Quess &, 5 & 4,2 & 2,1 &, 7 \\
\hline
\end{tabular}

Otra característica en la que coincide el presente estudio con otros publicados parecidos es en la gran variabilidad que hay entre las distintas aguas españolas. Así, en cuanto a contenido en calcio, el 50\% de las aguas contienen entre 25 y $85 \mathrm{mg} / \mathrm{l}$ (Fig. 1), con una media de $57,9 \mathrm{mg} / \mathrm{l}$ y un valor mínimo y máximo de 0,5 y $181 \mathrm{mg} / 1$, respectivamente (Tabla 1). El contenido en bicarbonato con una media de $295,2 \mathrm{mg} / \mathrm{l}$, un mínimo de $2,1 \mathrm{mg} / \mathrm{l}$ y un máximo de $2.196 \mathrm{mg} / \mathrm{l}$ (Tabla 1), tiene menos variabilidad ya que el contenido de bicarbonato del $50 \%$ de las aguas se mueve entre un estrecho rango entre 150 y 300 mg/l (Fig. 2). Sin embargo, existen 4 aguas con valores extremadamente altos: una superior a $1.000 \mathrm{mg} / 1$ (Vilajuiga) y 3 superiores a $2.000 \mathrm{mg} / 1$ (San Narciso, Malavella y Vichy Catalán) (Fig. 2 y Tabla 3). Como se comentará 
Tabla 3. Aguas embotelladas ordenadas en sentido descendente según el contenido en Bicarbonato (mg/l)

\begin{tabular}{|c|c|c|c|c|}
\hline Marca & Bicarbonato & $\mathbf{N a}$ & $\mathbf{C a}$ & Mg \\
\hline San Narciso & $2.196,0$ & $1.080,0$ & 51,4 & - \\
\hline Malavella & $2.147,0$ & $1.136,0$ & 48,9 & 8,1 \\
\hline Vichy Catalán & $2.081,0$ & $1.097,0$ & - & - \\
\hline Vilajuiga & $1.561,6$ & 568,0 & 83,4 & 46,7 \\
\hline Firgas & 513,8 & 62,9 & 79,7 & 41,4 \\
\hline Sierra de Cazorla & 465,4 & 1,3 & 77,8 & 37,1 \\
\hline Agua del Rosal & 400,2 & 7,2 & 85,7 & 33,5 \\
\hline Agua del Valle del Cardo & 400,2 & 7,2 & 85,7 & 33,5 \\
\hline Font del Cami & 379,6 & 39,3 & 87,4 & 43,3 \\
\hline Aquadeus & 345,5 & 3,7 & 80,2 & 30,5 \\
\hline Aigua mineral Bonpreu & 345,3 & 6,3 & 92,1 & 17,0 \\
\hline Sant Aniol & 343,1 & 6,0 & 92,1 & 15,4 \\
\hline Ribagorza & 331,8 & 25,3 & 69,8 & 25,2 \\
\hline Font del Pi & 316,6 & 26,9 & 66,5 & 65,6 \\
\hline Font Jaraba & 301,3 & 42,5 & 100,3 & 40,9 \\
\hline Fontecabras & 300,7 & ,0 & 93,0 & 38,9 \\
\hline Monte Pinos & 298,0 & 1,8 & 93,8 & 3,4 \\
\hline Monte Pinos & 298,0 & 1,8 & 93,8 & 3,4 \\
\hline Lunares & 296,3 & 39,5 & 102,7 & 36,7 \\
\hline Fuente Primavera & 295,3 & 20,7 & 86,6 & 23,3 \\
\hline Fontecelta & 288,3 & 89,5 & 26,5 & - \\
\hline Solan de Cabras & 285,0 & 5,3 & 56,9 & 25,5 \\
\hline Agua del Rosal & 280,3 & 49,8 & 66,2 & 11,1 \\
\hline Aquabona & 277,0 & 2,4 & 90,3 & 2,8 \\
\hline Na Taconera & 275,0 & 49,0 & 144,0 & 28,0 \\
\hline Font De Pla Nova & 270,0 & 11,0 & 74,0 & 36,0 \\
\hline Cortés & 268,4 & 6,4 & 81,9 & 7,2 \\
\hline Fuente Liviana & 268,3 & ,8 & 65,4 & 17,0 \\
\hline Marquesado & 267,7 &, 8 & 70,5 & 18,5 \\
\hline Benassal & 267,2 & 2,7 & 92,2 & ,0 \\
\hline Santolin & 266,0 & 2,6 & 89,8 & 2,4 \\
\hline El portell & 257,4 & 23,6 & 95,4 & 12,2 \\
\hline Font Agudes del Montseny & 252,2 & 41,3 & 53,8 & 14,3 \\
\hline Font Selva & 249,0 & 1,0 & 6,0 & 54,9 \\
\hline Solares & 248,9 & 87,6 & 73,7 & 15,6 \\
\hline Vitel & 248,0 & 7,7 & 94,0 & 20,0 \\
\hline Agua de Cuevas & 246,9 & 1,6 & 47,0 & 25,4 \\
\hline Font Teca & 244,0 & - & 115,0 & 51,0 \\
\hline Castro Vita & 240,3 & 8,6 & 76,5 & 16,1 \\
\hline San Pellegrino & 239,0 & 36,1 & 181,0 & 53,5 \\
\hline Agua de Serria Balnéa & 239,0 & - & 118,0 & 51,0 \\
\hline Agua de Sierra & 239,0 & - & 118,0 & 51,0 \\
\hline Pineo & 238,0 & 1,5 & 74,0 & 4,1 \\
\hline Fuente Solana & 226,3 & 19,2 & 67,1 & 18,0 \\
\hline Fuentedueñas & 226,0 & 5,6 & 59,6 & 8,4 \\
\hline Agua mineral Carrefour & 225,1 &, 8 & 64,1 & 18,5 \\
\hline
\end{tabular}


Tabla 3. (Continuación)

\begin{tabular}{|c|c|c|c|c|}
\hline Marca & $\mathbf{C a}$ & $\mathbf{N a}$ & Bicarbonato & Mg \\
\hline Fuensanta & 222,2 & 15,9 & 71,3 & 10,9 \\
\hline Agua mineral Auchan & 213,8 & 49,7 & 33,9 & 5,0 \\
\hline Fonteforte & 205,6 & 108,5 & 80,6 & 19,9 \\
\hline Azuébar & 200,0 & 7,7 & 28,5 & - \\
\hline Veri & 197,0 &, 6 & 69,0 & 1,5 \\
\hline Agua Alzola & 192,0 & 44,0 & 44,0 & 5,8 \\
\hline Valtorre & 191,0 & 30,5 & 25,6 & 23,6 \\
\hline Aguavida & 171,0 & 7,2 & 2,8 & 57,1 \\
\hline Fondalt & 163,6 & 5,0 & 50,5 & 15,8 \\
\hline Agua de Ribes & 163,5 & 4,9 & 54,4 & 7,5 \\
\hline Mondariz & 163,4 & 50,5 & 9,2 & 4,9 \\
\hline Cabreiroá & 162,3 & 49,3 & 9,5 & 4,5 \\
\hline Insalus & 156,4 & 10,0 & 157,3 & 19,3 \\
\hline Agua San Joaquín & 152,3 & 10,4 & 41,2 & 12,3 \\
\hline Font Vella & 149,0 & 13,2 & 38,5 & 9,7 \\
\hline Pallars & 140,3 & 67,0 & 62,5 & 22,4 \\
\hline Sierres de Jaen & 135,0 & 1,8 & 36,0 & 15,0 \\
\hline Font del Regàs & 133,1 & 14,7 & 36,2 & 3,4 \\
\hline Fonter & 127,0 & 7,4 & 32,0 & 7,3 \\
\hline Les Creus & 119,0 & 11,7 & 28,0 & 7,3 \\
\hline Aquarel & 115,0 & 10,0 & 34,0 & 4,0 \\
\hline Calabor & 115,0 & 47,7 & 5,1 &, 9 \\
\hline Viladrau & 112,3 & 9,6 & 27,0 & 4,8 \\
\hline Agua de Borines & 105,8 & 35,3 & 9,6 & 2,9 \\
\hline Lanjarón & 105,0 & 4,8 & 27,2 & 8,8 \\
\hline Carrizal & 104,0 & 1,0 & 27,0 & 6,0 \\
\hline San Vicente & 81,1 & 5,9 & 22,0 & 7,3 \\
\hline Sousas & 79,8 & 29,0 & 3,4 & ,9 \\
\hline Font d`Or & 78,8 & 9,2 & 26,4 & 3,2 \\
\hline La Platina & 78,7 & 9,4 & 16,0 & 15,8 \\
\hline Fonteide & 57,1 & 19,1 & 6,4 & 3,7 \\
\hline Aigua del Montseny & 48,7 & 9,8 & 13,1 & 4,2 \\
\hline Caldes de Boí & 38,7 & 39,2 & 7,3 &, 7 \\
\hline Fonsana & 38,0 & 7,0 & 5,3 & ,9 \\
\hline San Carlos & 21,0 & 31,0 & 12,0 & 4,3 \\
\hline Bezoya & 6,7 & 2,3 & 1,6 &, 3 \\
\hline Agua sana & 4,6 & 5,9 &, 5 & ,8 \\
\hline Quess & 2,1 & 4,2 &, 5 &, 7 \\
\hline Fontanel & - & 39,6 & 36,3 & 10,9 \\
\hline
\end{tabular}

más adelante, este hecho tiene importancia para el tratamiento de las litiasis úricas. Todo lo comentado con el bicarbonato se puede aplicar al sodio, ya que con una media de $67,3 \mathrm{mg} / 1$ (Tabla 1) presenta poca variabilidad, pero existen 4 casos de aguas con valores extremadamente altos (Fig. 3 y Tabla 4) que coinciden con las 4 marcas de agua con un alto con- tenido en bicarbonato. Esto se debe a la fuerte correlación existente entre la concentración de bicarbonato y de sodio (Tabla 6). Como se comentará más adelante, este hecho comportará el principal problema del tratamiento de la litiasis úrica con aguas bicarbonatadas, sobre todo en pacientes hipertensos. La concentración de magnesio con una 
Tabla 4. Aguas embotelladas ordenadas en sentido descendente según el contenido en Sodio(mg/l)

\begin{tabular}{|c|c|c|c|c|}
\hline Marca & $\mathbf{N a}$ & Bicarbonato & $\mathbf{C a}$ & Mg \\
\hline Malavella & $1.136,0$ & $2.147,0$ & 48,9 & 8,1 \\
\hline VichyCatalán & $1.097,0$ & $2.081,0$ & - & - \\
\hline SanNarciso & $1.080,0$ & $2.196,0$ & 51,4 & - \\
\hline Vilajuiga & 568,0 & $1.561,6$ & 83,4 & 46,7 \\
\hline Fonteforte & 108,5 & 205,6 & 80,6 & 19,9 \\
\hline Fontecelta & 89,5 & 288,3 & 26,5 & ,0 \\
\hline Solares & 87,6 & 248,9 & 73,7 & 15,6 \\
\hline Pallars & 67,0 & 140,3 & 62,5 & 22,4 \\
\hline Firgas & 62,9 & 513,8 & 79,7 & 41,4 \\
\hline Mondariz & 50,5 & 163,4 & 9,2 & 4,9 \\
\hline AguadelRosal & 49,8 & 280,3 & 66,2 & 11,1 \\
\hline AguamineralAuchan & 49,7 & 213,8 & 33,9 & 5,0 \\
\hline Cabreiroá & 49,3 & 162,3 & 9,5 & 4,5 \\
\hline NaTaconera & 49,0 & 275,0 & 144,0 & 28,0 \\
\hline Calabor & 47,7 & 115,0 & 5,1 &, 9 \\
\hline AguaAlzola & 44,0 & 192,0 & 44,0 & 5,8 \\
\hline FontJaraba & 42,5 & 301,3 & 100,3 & 40,9 \\
\hline FontAgudesdelMontseny & 41,3 & 252,2 & 53,8 & 14,3 \\
\hline Fontanel & 39,6 & - & 36,3 & 10,9 \\
\hline Lunares & 39,5 & 296,3 & 102,7 & 36,7 \\
\hline FontdelCami & 39,3 & 379,6 & 87,4 & 43,3 \\
\hline CaldesdeBoí & 39,2 & 38,7 & 7,3 &, 7 \\
\hline SanPellegrino & 36,1 & 239,0 & 181,0 & 53,5 \\
\hline AguadeBorines & 35,3 & 105,8 & 9,6 & 2,9 \\
\hline SanCarlos & 31,0 & 21,0 & 12,0 & 4,3 \\
\hline Valtorre & 30,5 & 191,0 & 25,6 & 23,6 \\
\hline Sousas & 29,0 & 79,8 & 3,4 &, 9 \\
\hline FontdelPi & 26,9 & 316,6 & 66,5 & 65,6 \\
\hline Ribagorza & 25,3 & 331,8 & 69,8 & 25,2 \\
\hline Elportell & 23,6 & 257,4 & 95,4 & 12,2 \\
\hline FuentePrimavera & 20,7 & 295,3 & 86,6 & 23,3 \\
\hline FuenteSolana & 19,2 & 226,3 & 67,1 & 18,0 \\
\hline Fonteide & 19,1 & 57,1 & 6,4 & 3,7 \\
\hline Fuensanta & 15,9 & 222,2 & 71,3 & 10,9 \\
\hline FontdelRegàs & 14,7 & 133,1 & 36,2 & 3,4 \\
\hline FontVella & 13,2 & 149,0 & 38,5 & 9,7 \\
\hline LesCreus & 11,7 & 119,0 & 28,0 & 7,3 \\
\hline FontDePlaNova & 11,0 & 270,0 & 74,0 & 36,0 \\
\hline AguaSanJoaquín & 10,4 & 152,3 & 41,2 & 12,3 \\
\hline Insalus & 10,0 & 156,4 & 157,3 & 19,3 \\
\hline Aquarel & 10,0 & 115,0 & 34,0 & 4,0 \\
\hline AiguadelMontseny & 9,8 & 48,7 & 13,1 & 4,2 \\
\hline Viladrau & 9,6 & 112,3 & 27,0 & 4,8 \\
\hline LaPlatina & 9,4 & 78,7 & 16,0 & 15,8 \\
\hline Fontd`Or & 9,2 & 78,8 & 26,4 & 3,2 \\
\hline CastroVita & 8,6 & 240,3 & 76,5 & 16,1 \\
\hline
\end{tabular}


Tabla 4. (Continuación)

\begin{tabular}{|c|c|c|c|c|}
\hline Marca & $\mathbf{N a}$ & Bicarbonato & $\mathbf{C a}$ & Mg \\
\hline Azuébar & 7,7 & 200,0 & 28,5 & 0 \\
\hline Vitel & 7,7 & 248,0 & 94,0 & 20,0 \\
\hline Fonter & 7,4 & 127,0 & 32,0 & 7,3 \\
\hline AguadelRosal & 7,2 & 400,2 & 85,7 & 33,5 \\
\hline Aguavida & 7,2 & 171,0 & 2,8 & 57,1 \\
\hline AguadelValledelCardo & 7,2 & 400,2 & 85,7 & 33,5 \\
\hline Fonsana & 7,0 & 38,0 & 5,3 &, 9 \\
\hline Cortés & 6,4 & 268,4 & 81,9 & 7,2 \\
\hline AiguamineralBonpreu & 6,3 & 345,3 & 92,1 & 17,0 \\
\hline SantAniol & 6,0 & 343,1 & 92,1 & 15,4 \\
\hline Aguasana & 5,9 & 4,6 &, 5 & ,8 \\
\hline SanVicente & 5,9 & 81,1 & 22,0 & 7,3 \\
\hline Fuentedueñas & 5,6 & 226,0 & 59,6 & 8,4 \\
\hline SolandeCabras & 5,3 & 285,0 & 56,9 & 25,5 \\
\hline Fondalt & 5,0 & 163,6 & 50,5 & 15,8 \\
\hline AguadeRibes & 4,9 & 163,5 & 54,4 & 7,5 \\
\hline Lanjarón & 4,8 & 105,0 & 27,2 & 8,8 \\
\hline Quess & 4,2 & 2,1 &, 5 &, 7 \\
\hline Aquadeus & 3,7 & 345,5 & 80,2 & 30,5 \\
\hline Benassal & 2,7 & 267,2 & 92,2 &, 0 \\
\hline Santolin & 2,6 & 266,0 & 89,8 & 2,4 \\
\hline Aquabona & 2,4 & 277,0 & 90,3 & 2,8 \\
\hline Bezoya & 2,3 & 6,7 & 1,6 &, 3 \\
\hline MontePinos & 1,8 & 298,0 & 93,8 & 3,4 \\
\hline SierresdeJaen & 1,8 & 135,0 & 36,0 & 15,0 \\
\hline AguadeCuevas & 1,6 & 246,9 & 47,0 & 25,4 \\
\hline Pineo & 1,5 & 238,0 & 74,0 & 4,1 \\
\hline SierradeCazorla & 1,3 & 465,4 & 77,8 & 37,1 \\
\hline FontSelva & 1,0 & 249,0 & 6,0 & 54,9 \\
\hline Carrizal & 1,0 & 104,0 & 27,0 & 6,0 \\
\hline FuenteLiviana & ,8 & 268,3 & 65,4 & 17,0 \\
\hline AguamineralCarrefour & ,8 & 225,1 & 64,1 & 18,5 \\
\hline Marquesado & ,8 & 267,7 & 70,5 & 18,5 \\
\hline Veri & ,6 & 197,0 & 69,0 & 1,5 \\
\hline Fontecabras & - & 300,7 & 93,0 & 38,9 \\
\hline AguadeSerriaBalnéa & - & 239,0 & 118,0 & 51,0 \\
\hline AguadeSierra & - & 239,0 & 118,0 & 51,0 \\
\hline FontTeca & - & 244,0 & 115,0 & 51,0 \\
\hline
\end{tabular}

media de $18 \mathrm{mg} / 1$ (Tabla 1) sigue una distribución parecida a la del calcio (Fig. 4), con el que tiene una correlación estadísticamente significativa aunque no tan intensa como la existente entre bicarbonato y sodio (Tabla 6).

Otro hallazgo interesante de Martín-Gil et $\mathrm{al}^{24}$ fue la variabilidad de un mismo tipo de agua. Así, encontraron aguas que han ido variando su compo- sición en los últimos años. Este hecho, lo hemos encontrado también en nuestro estudio, en algún caso entre etiquetas de la misma marca de agua. Debido a esto, es importante que la información del análisis químico de las aguas de nuestro trabajo, vaya siendo actualizada periódicamente por cada urólogo, entre las aguas de grifo o embotelladas de mayor uso en su región. 
Tabla 5. Aguas embotelladas ordenadas en sentido descendente según el contenido en Magnesio (mg/l)

\begin{tabular}{|c|c|c|c|c|}
\hline Marca & Mg & $\mathbf{C a}$ & $\mathbf{N a}$ & Bicarbonato \\
\hline Font del Pi & 65,6 & 66,5 & 26,9 & 316,6 \\
\hline Aguavida & 57,1 & 2,8 & 7,2 & 171,0 \\
\hline Font Selva & 54,9 & 6,0 & 1,0 & 249,0 \\
\hline San Pellegrino & 53,5 & 181,0 & 36,1 & 239,0 \\
\hline Agua de Serria Balnéa & 51,0 & 118,0 & - & 239,0 \\
\hline Agua de Sierra & 51,0 & 118,0 & - & 239,0 \\
\hline Font Teca & 51,0 & 115,0 & - & 244,0 \\
\hline Vilajuiga & 46,7 & 83,4 & 568,0 & 1561,6 \\
\hline Font del Cami & 43,3 & 87,4 & 39,3 & 379,6 \\
\hline Firgas & 41,4 & 79,7 & 62,9 & 513,8 \\
\hline Font Jaraba & 40,9 & 100,3 & 42,5 & 301,3 \\
\hline Fontecabras & 38,9 & 93,0 & - & 300,7 \\
\hline Sierra de Cazorla & 37,1 & 77,8 & 1,3 & 465,4 \\
\hline Lunares & 36,7 & 102,7 & 39,5 & 296,3 \\
\hline Font De Pla Nova & 36,0 & 74,0 & 11,0 & 270,0 \\
\hline Agua del Rosal & 33,5 & 85,7 & 7,2 & 400,2 \\
\hline Agua del Valle del Cardo & 33,5 & 85,7 & 7,2 & 400,2 \\
\hline Aquadeus & 30,5 & 80,2 & 3,7 & 345,5 \\
\hline Na Taconera & 28,0 & 144,0 & 49,0 & 275,0 \\
\hline Solan de Cabras & 25,5 & 56,9 & 5,3 & 285,0 \\
\hline Agua de Cuevas & 25,4 & 47,0 & 1,6 & 246,9 \\
\hline Ribagorza & 25,2 & 69,8 & 25,3 & 331,8 \\
\hline Valtorre & 23,6 & 25,6 & 30,5 & 191,0 \\
\hline Fuente Primavera & 23,3 & 86,6 & 20,7 & 295,3 \\
\hline Pallars & 22,4 & 62,5 & 67,0 & 140,3 \\
\hline Vitel & 20,0 & 94,0 & 7,7 & 248,0 \\
\hline Fonteforte & 19,9 & 80,6 & 108,5 & 205,6 \\
\hline Insalus & 19,3 & 157,3 & 10,0 & 156,4 \\
\hline Agua mineral Carrefour & 18,5 & 64,1 &, 8 & 225,1 \\
\hline Marquesado & 18,5 & 70,5 & ,8 & 267,7 \\
\hline Fuente Solana & 18,0 & 67,1 & 19,2 & 226,3 \\
\hline Fuente Liviana & 17,0 & 65,4 & ,8 & 268,3 \\
\hline Aigua mineral Bonpreu & 17,0 & 92,1 & 6,3 & 345,3 \\
\hline Castro Vita & 16,1 & 76,5 & 8,6 & 240,3 \\
\hline Fondalt & 15,8 & 50,5 & 5,0 & 163,6 \\
\hline La Platina & 15,8 & 16,0 & 9,4 & 78,7 \\
\hline Solares & 15,6 & 73,7 & 87,6 & 248,9 \\
\hline Sant Aniol & 15,4 & 92,1 & 6,0 & 343,1 \\
\hline Sierres de Jaen & 15,0 & 36,0 & 1,8 & 135,0 \\
\hline Font Agudes del Montseny & 14,3 & 53,8 & 41,3 & 252,2 \\
\hline Agua San Joaquín & 12,3 & 41,2 & 10,4 & 152,3 \\
\hline $\mathrm{El}$ portell & 12,2 & 95,4 & 23,6 & 257,4 \\
\hline Agua del Rosal & 11,1 & 66,2 & 49,8 & 280,3 \\
\hline Fuensanta & 10,9 & 71,3 & 15,9 & 222,2 \\
\hline Fontanel & 10,9 & 36,3 & 39,6 & - \\
\hline
\end{tabular}


Tabla 5. (Continuación)

\begin{tabular}{|c|c|c|c|c|}
\hline Marca & Mg & $\mathbf{C a}$ & $\mathbf{N a}$ & Bicarbonato \\
\hline Font Vella & 9,7 & 38,5 & 13,2 & 149,0 \\
\hline Lanjarón & 8,8 & 27,2 & 4,8 & 105,0 \\
\hline Fuentedueñas & 8,4 & 59,6 & 5,6 & 226,0 \\
\hline Malavella & 8,1 & 48,9 & $1.136,0$ & $2.147,0$ \\
\hline Agua de Ribes & 7,5 & 54,4 & 4,9 & 163,5 \\
\hline Les Creus & 7,3 & 28,0 & 11,7 & 119,0 \\
\hline Fonter & 7,3 & 32,0 & 7,4 & 127,0 \\
\hline San Vicente & 7,3 & 22,0 & 5,9 & 81,1 \\
\hline Cortés & 7,2 & 81,9 & 6,4 & 268,4 \\
\hline Carrizal & 6,0 & 27,0 & 1,0 & 104,0 \\
\hline Agua Alzola & 5,8 & 44,0 & 44,0 & 192,0 \\
\hline Agua mineral Auchan & 5,0 & 33,9 & 49,7 & 213,8 \\
\hline Mondariz & 4,9 & 9,2 & 50,5 & 163,4 \\
\hline Viladrau & 4,8 & 27,0 & 9,6 & 112,3 \\
\hline Cabreiroá & 4,5 & 9,5 & 49,3 & 162,3 \\
\hline San Carlos & 4,3 & 12,0 & 31,0 & 21,0 \\
\hline Aigua del Montseny & 4,2 & 13,1 & 9,8 & 48,7 \\
\hline Pineo & 4,1 & 74,0 & 1,5 & 238,0 \\
\hline Aquarel & 4,0 & 34,0 & 10,0 & 115,0 \\
\hline Fonteide & 3,7 & 6,4 & 19,1 & 57,1 \\
\hline Font del Regàs & 3,4 & 36,2 & 14,7 & 133,1 \\
\hline Monte Pinos & 3,4 & 93,8 & 1,8 & 298,0 \\
\hline Font d`or & 3,2 & 26,4 & 9,2 & 78,8 \\
\hline Agua de Borines & 2,9 & 9,6 & 35,3 & 105,8 \\
\hline Aquabona & 2,8 & 90,3 & 2,4 & 277,0 \\
\hline Santolin & 2,4 & 89,8 & 2,6 & 266,0 \\
\hline Veri & 1,5 & 69,0 & ,6 & 197,0 \\
\hline Calabor &, 9 & 5,1 & 47,7 & 115,0 \\
\hline Sousas &, 9 & 3,4 & 29,0 & 79,8 \\
\hline Fonsana & ,9 & 5,3 & 7,0 & 38,0 \\
\hline Agua sana & ,8 &, 5 & 5,9 & 4,6 \\
\hline Caldes de Boí &, 7 & 7,3 & 39,2 & 38,7 \\
\hline Quess &, 7 &, 5 & 4,2 & 2,1 \\
\hline Bezoya & ,3 & 1,6 & 2,3 & 6,7 \\
\hline Azuébar & - & 28,5 & 7,7 & 200,0 \\
\hline Fontecelta & - & 26,5 & 89,5 & 288,3 \\
\hline Vichy Catalán & - & ,0 & $1.097,0$ & $2.081,0$ \\
\hline Benassal & - & 92,2 & 2,7 & 267,2 \\
\hline San Narciso & - & 51,4 & $1.080,0$ & $2.196,0$ \\
\hline
\end{tabular}

Aunque no hemos obtenido información directa sobre los sistemas Brita y Grupo Corsa, existe una publicación que entre otras cosas evaluó la eficacia del primero ${ }^{11}$. De este modo, analizó la acción del uso consecutivo de 3 filtros Brita nuevos sobre la composición de 7 botellas de agua de marca Perrier. Encontraron dos cosas: una disminución media del calcio de un $89.4 \%$ y una gran variabilidad de eficacia entre los filtros. Además en todos los casos eran filtros nuevos, por lo que no se pudo evaluar su acción tras un uso repetitivo. A falta de más información o más estudios sobre el efecto del filtro Brita, hemos de considerar que su uso va a producir una disminución impredecible de la concentración de calcio. 
Tabla 6. Correlación entre los principales elementos químicos de las Aguas Embotelladas. Coeficiente de Correlación de Pearson (Significación estadística)

\begin{tabular}{lcccc}
\hline & Calcio & Bicarbonato & Sodio & Magnesio \\
\hline Calcio & - & $0,2(\mathrm{p}=0,07)$ & $0,013(\mathrm{p}=0,91)$ & $0,503(\mathrm{p}=0,0001)$ \\
Bicarbonato & $0,2(\mathrm{p}=0,07)$ & - & $0,958(\mathrm{p}=0,0001)$ & $0,264(\mathrm{p}=0,019)$ \\
Sodio & $0,013(\mathrm{p}=0,91)$ & $0,958(\mathrm{p}=0,0001)$ & - & $0,061(\mathrm{p}=0,599)$ \\
Magnesio & $0,503(\mathrm{p}=0,0001)$ & $0,264(\mathrm{p}=0,019)$ & $0,061(\mathrm{p}=0,599)$ & - \\
\hline
\end{tabular}

Tabla 7. Contenido en Calcio (mg/l) de las aguas de grifo de las capitales españolas

\begin{tabular}{|c|c|c|c|c|c|c|c|c|}
\hline Capital & 1 & 2 & Capital & 1 & 2 & Capital & 1 & 2 \\
\hline Albacete & 139,6 & - & Girona & 57,6 & 57,6 & Pamplona & 45,6 & 45,8 \\
\hline Alicante & 56,5 & 64,0 & Granada & 40 & $30-50$ & S Sebastián & - & - \\
\hline Almería & 59,2 & - & Huelva & 13,3 & 13,3 & Santander & - & - \\
\hline Ávila & $<15$ & - & Huesca & 74 & 74 & Segovia & $<15$ & - \\
\hline Badajoz & 45 & - & Jaén & 85,5 & - & Sevilla & 33 & - \\
\hline BCN Ter & 57,7 & 56 & León & 35 & - & Tarragona & 108 & 108 \\
\hline BCN Llobre & 127,9 & 115 & Lleida & 51,2 & 51,2 & SC Tenerife & 31 & - \\
\hline Bilbao & 36,7 & - & Logroño & 48 & 48 & Teruel & 104 & 104 \\
\hline Burgos & 15,8 & - & Madrid & $5,8-34,8$ & $2,9-41$ & Valencia & 121 & 121 \\
\hline Cádiz & 82,2 & 82,2 & Málaga & 25 & 76 & Valladolid & 160 & - \\
\hline Coruña & 16 & - & Murcia & 25,4 & - & Vitoria & $45-54$ & - \\
\hline Castellón & 131 & 131 & Orense & 28 & - & Zamora & 70 & - \\
\hline Ciudad Real & 40 & - & Oviedo & 61 & - & Zaragoza & 106 & 106 \\
\hline Córdoba & 11 & 11 & Palma & 78 & - & & & \\
\hline
\end{tabular}

1. Martínez-Ferrer y col'12 2. Vitoria y $\operatorname{Arias}^{23}$

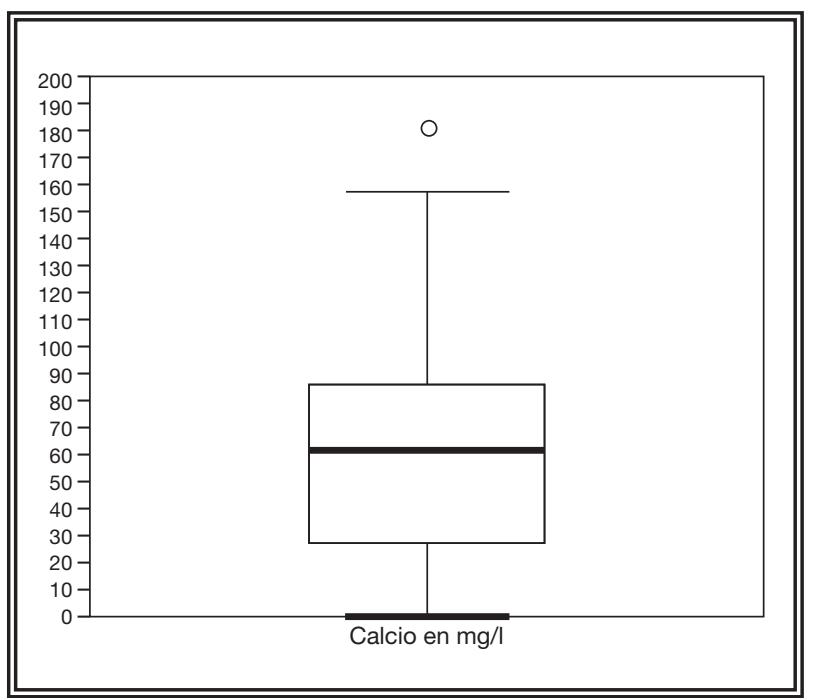

FIGURA 1. Distribución de la concentración de calcio (mg/l) en 85 aguas embotelladas.

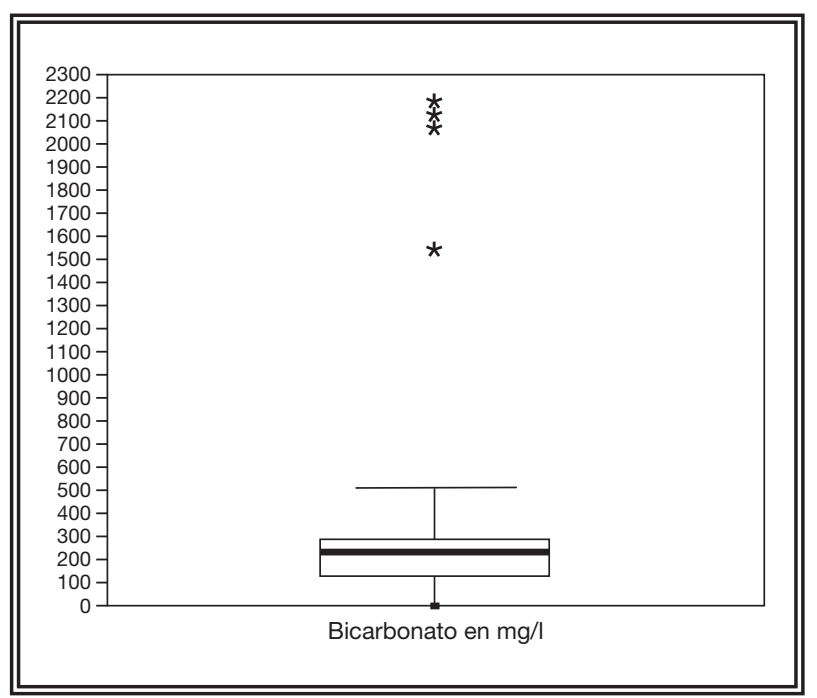

FIGURA 2. Distribución de la concentración de bicarbonato (mg/l) en 85 aguas embotelladas. 


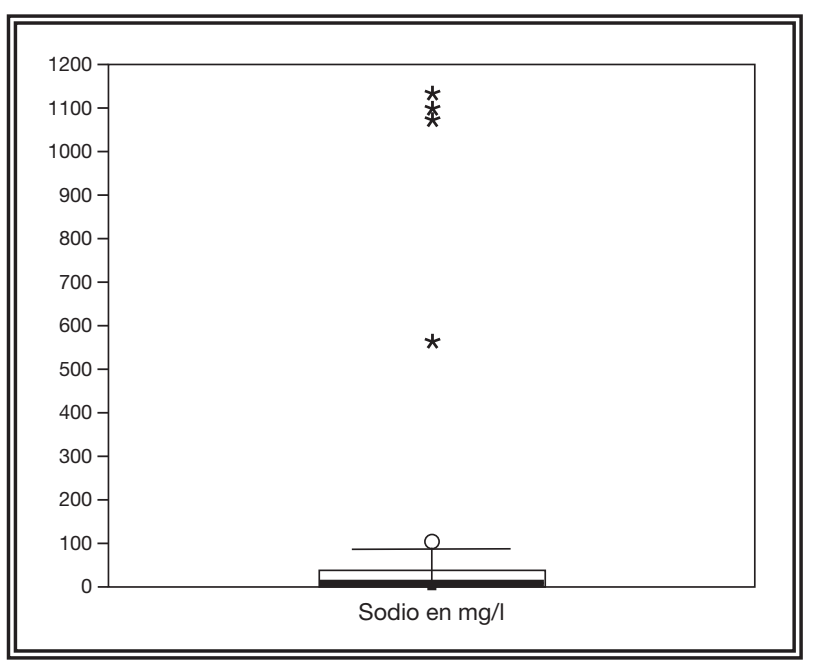

FIGURA 3. Distribución de la concentración de sodio (mg/l) en 85 aguas embotelladas.

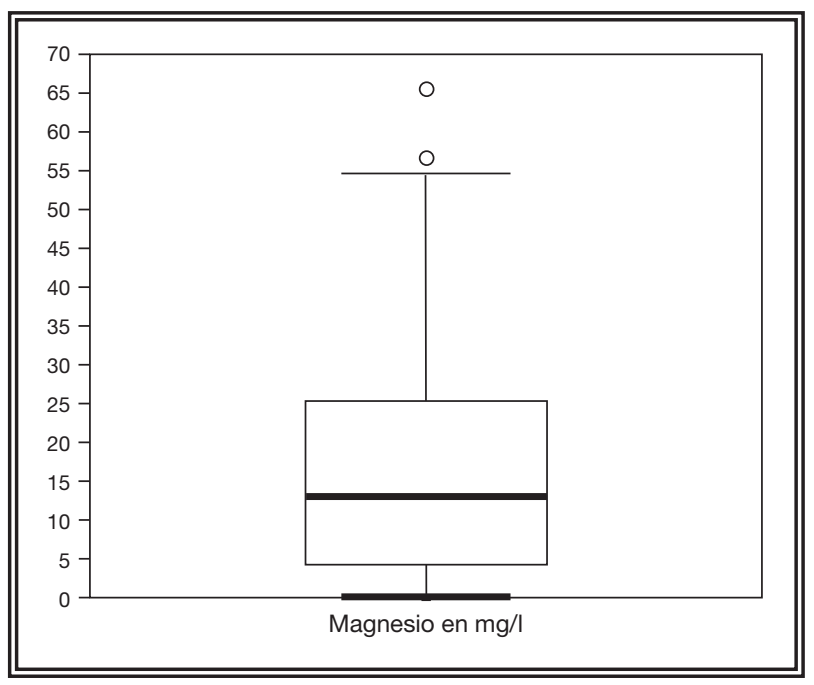

FIGURA 4. Distribución de la concentración de magnesio (mg/l) en 85 aguas embotelladas.

\section{¿Qué tipo de agua debe tomar un paciente litiásico? Propuesta terapéutica}

Hasta ahora se ha hablado de los diferentes tipos de aguas de grifo y embotellada existentes en nuestro país, de sus diferencias en cuanto a su composición química y de su variabilidad. Es ahora el momento de responder a la pregunta que nos hace todo paciente litiásico ¿qué tipo de agua he de tomar? En primer lugar, la primera recomendación a un paciente litiásico debe ser que aumente su ingesta líquida hasta conseguir unas diuresis de $2.000 \mathrm{ml}$, por lo que la ingesta debería ser como mínimo de estos $2.000 \mathrm{ml}$, variando en función del peso, la actividad y las características meteorológicas. Esta simple medida ya se ha comentado que es útil para disminuir la recurrencia litiásica ${ }^{4}$, y entre otras cosas, va a contribuir a también a disminuir la osmolaridad y la concentración de sodio en orina que se recomienda que sean inferiores a $1.020 \mathrm{~g} / \mathrm{cm}^{3}$ y $200 \mathrm{mmol} / 24 \mathrm{~h}$, respectivamente.

Una vez aceptado que a mayor ingesta de agua, menor riesgo de recurrencia litiásica, el tema de qué agua es mejor tomar, no dependerá de si es del grifo o embotellada, sino de su composición química sobre todo en lo referente al calcio. Lo determinante en la formación de la litiasis es la ingesta total de calcio diaria provenga de donde provenga (agua, alimentos o lácteos). El aporte diario de calcio de un adulto ha de ser de $1.000 \mathrm{mg}$; si con la ingesta de productos no lácteos se consigue un aporte de $200-300 \mathrm{mg}$, quedan 700-800 mg que deben ser aportados entre la toma de productos lácteos y la ingesta de agua (Tabla 8). Es por ello, que al igual que otros autores ${ }^{25}$ en nuestro grupo realizamos recomendaciones individualizadas según los hábitos de toma de lácteos que realiza cada paciente. La regla general sería recomendar aguas bajas en calcio a los pacientes que toman abundantes lácteos (sobre todo quesos), y por el contrario, tomar aguas ricas en calcio en aquellos casos en que se tomen pocos lácteos. Hay que tener en cuenta que con la toma diaria de 100 $\mathrm{g}$ de algunos tipos de queso (Roquefort, Mozzarella, Gorgonzola, azules) se consigue la dosis diaria recomendada de calcio, o incluso se supera (Edam, Gouda, Parmesano, Emmental, Gruyère).

Por otro lado, con una ingesta de $2.500 \mathrm{ml} \mathrm{de}$ agua de alto contenido en calcio (San Pellegrino, Insalus, Na Taconera, Serria Balneá, Agua de Sierra, Font Teca o las del grifo de Albacete, Barcelona Llobregat, Tarragona, Teruel, Valencia, Valladolid o Zaragoza) se consigue un aporte de unos 250-450 mg diarios de calcio, lo que sumado a los 200- $300 \mathrm{mg}$ de calcio aportados por los productos no lácteos, significa que sólo habría que aportar mediante el consumo de productos lácteos unos 250-550 mg de calcio. Por ello, si se toma este tipo de aguas no estaría recomendado tomar queso que no fuera fresco o tipo Brie. Por el contrario, aquellas personas que tomen quesos con alto contenido en calcio, deberían tomar aguas de bajo contenido en calcio. Si beben agua del grifo y viven en ciudades como Ávila, Badajoz, Bilbao, Burgos, A Coruña, Ciudad Real, Córdoba, Granada, Huelva, León, Logroño, Madrid, Málaga, Murcia, Pamplona, Segovia, Sevilla o Santa Cruz de Tenerife, no sería ningún problema porque con una ingesta de 2 litros 
Tabla 8. Aporte de calcio en mg de diferentes alimentos

$\begin{array}{ll}\begin{array}{l}\text { Productos no lácteos } \\ 200-300 \mathrm{mg} / \text { dia }\end{array} & \text { Quesos (por cada } 100 \mathrm{mg}) \\ \begin{array}{l}\text { Aguas embotelladas } \\ \text { Ver Tabla } 2\end{array} & \text { Frescos, Brie, } 120 \mathrm{mg} \\ \begin{array}{l}\text { Aguas del grifo } \\ \text { Ver Tabla } 7\end{array} & \text { Camembert } 400 \mathrm{mg} \\ \begin{array}{l}\text { Leche } 100 \mathrm{ml} 120 \mathrm{mg} \\ \text { Yogurt (1 unidad = } 125 \mathrm{~g})\end{array} & \text { Mozzarella, Gorgonzola, azules } 700 \mathrm{mg} \\ 150-200 \mathrm{mg} & \text { Edam, Gorda } 1.000 \mathrm{mg} \\ \text { Petit Suisse (100 mg) } 120 \mathrm{mg} & \text { Parmesano, Emmental, Gruyère } 1.200 \mathrm{mg} \\ & \begin{array}{l}\text { El aporte total diario de calcio ha de ser de } \\ 1.000 \text { mg. }\end{array}\end{array}$

nacional y a la provincia de Albacete, respectivamente. Aunque el presente trabajo analiza un número superior de marcas de agua y relaciona su consumo dentro del contexto general del consumo del calcio en la dieta, existe entre todos un denominador común: el llamar la atención al urólogo que trata paciente litiásicos, sobre la necesidad de conocer las características del agua del grifo de su localidad y de las marcas comerciales que beben sus pacientes, para poder realizar unas recomen-

diarios estarán aportando menos de 100 mg de calcio. Y si estas personas consumidoras de queso con alto contenido en calcio viven en otras ciudades o no quieren tomar agua del grifo, tal como recoge la Tabla 3, hay 36 marcas con concentraciones inferiores a $50 \mathrm{mg} / \mathrm{l}$, e incluso 16 con valores inferiores a $20 \mathrm{mg} / 1$.

En cuanto a la litiasis úrica, de todos es sabido que su tratamiento se ve favorecido con la alcalinización de la orina. Ésta se puede conseguir mediante la toma de aguas bicarbonatadas, pero debido a la gran correlación que hay entre la concentración de bicarbonato y sodio (Tabla 6), y dado que la ingesta de sodio aumenta la excreción renal de calcio, a la larga puede suponer un problema para el paciente. Así, tomando 21 diarios de las aguas más bicarbonatadas (San Narciso, Malavella, y Vichy Catalán) (Tabla 3) se estarían ingiriendo los 2 g de sodio máximos diarios, por lo que se debería realizar una dieta asódica total, cosa extremadamente difícil de llevar a cabo. Una opción podría ser tomar cualquiera de estas 3 marcas de agua durante períodos cortos de tiempo y siempre que el paciente no sea hipertenso. De lo contrario, se podría tomar aguas con un contenido moderado de bicarbonato pero muy bajo de sodio (Firgas, Sierra de Cazorla, Agua del Rosal, Agua del Valle del Cardo, Font del Cami, Aquadeus, Bonpreu o Sant Aniol) (Tabla 3). Por otro lado, aquellos pacientes afectos de litiasis oxalocálcica con orinas ácidas también podrían beneficiarse de la ingesta de aguas con un contenido moderado en bicarbonato y calcio y bajo en sodio.

Finalmente, existen otros trabajos previos publicados con objetivos similares como los de Vallejo et al. ${ }^{26}$ y Alapont et al. ${ }^{27}$ circunscritos a todo el territorio daciones individualizadas en función del perfil y de las preferencias dietéticas del paciente.

\section{REFERENCIAS}

1. Petraccia L, Liberati G, Masciullo SG, Grassi M, Fraioli A. Water, mineral waters and health. Clinical Nutrition. 2006;25 (3):377-385.

2. Azoulay A, Garzon P, Eisenbert MJ. Comparison of the mineral content of tap water and bottled waters. J Gen Intern Med. 2001;16(3):168-175.

3. Borghi L, Meschi T, Amato F, Briganti A, Novarini A, Giannini A. Urinary volume, water and recurrences in idiopathic calcium nephrolithiasis: a 5-year randomized prospective study. J Urol. 1996;155(3):839-853.

4. Curhan GC, Willett WC, Rimm EB, Stampfer MJ. A prospective study of dietary calcium and other nutrients and the risk of symptomatic kidney stones. N Engl J Med. 1993;328(12):833838.

5. Garcia Matilla F, Garcia Montes F, Ribas Serna J. Diuresis acuosa y profilaxis de la nefrolitiasis recidivante. Actas Urol Esp. 1999;23(4):296-308.

6. Garcia Matilla F, Garcia Montes F, Ribas Serna J. Relaciones entre diuresis, $\mathrm{pH}$ de la orina y litogénesis. Actas Urol Esp. 1999;23(3):202-213.

7. Medina López RA, Ribas Serna J, Garcia Matilla F. Efectos de la diuresis acuosa sobre el $\mathrm{pH}$ urinario de los pacientes litiásicos recidivantes. Actas Urol Esp. 2003;27(5):361-369.

8. Borghi L, Schianchi T, Meschi T, Guerra A, Allegri F, Maggiore $\mathrm{U}$, Novarini A. Comparison of two diets for the prevention of recurrent stones in idiopathic hypercalciuria. N Engl J Med. 2002. 346(2):77-84.

9. Curhan GC, Willett WC, Speizer FE, Spiegelman D, Stampfer MJ. Comparison of dietary calcium with supplemental calcium and other nutrients as factors affecting the risk for kidney stones in women. Ann Intern Med. 1997;126(7):497-504.

10. Borsatti A. Calcium oxalate nephrolithiasis: defective oxalate transport. Kidney Int. 1991;39(6):1283-1298.

11. Morr S, Cuartas E, Alwasttar B, Lane JM. How much calcium is in your drinking water? A survey of calcium concentrations in bottled and tap waster and their significance for medical treatment and drug administration. HSSJ. 2006;2:130-135.

12. Martínez-Ferrer A, Peris P, Reyes R, Guañabens N. Aporte de calcio, magnesio y sodio a través del agua embotellada y de las aguas de consumo público: implicaciones para la salud. Med Clin. 2008;131(17):641-646. 
13. Caudarella R, Rizzoli E, Buffa A, Obtura A, Stefoni S. Comparative study of the influence of 3 types of mineral water in patients with idiopathic calcium lithiasis. J Urol. 1998;159 (3):658-663.

14. Rodgers AL. Effect of mineral water containing calcium and magnesium on calcium oxalate urolithiasis risk factors. Urol Int. 1997;58(2):93-99.

15. Coen G, Sasrdella D, Barbera G, Ferrannini M, Comegna C, Ferazolli F, Dinnella A, D’Anello E, Simeoni P. Urinary composition and lithogenic risk in normal subjects following oligomineral versus bicarbonate-alkaline high calcium mineral water intake. Urol Int. 2001;67(1):49-53.

16. Karagülle O, Smorag U, Candir F, Gundermann G, Jonas U, Becker AJ, Gehrke A, Gutenbrunner C. Clinical study on the effect of mineral waters containing bicarbonate on the risk of urinary stone formation in patients with multiple episodes of CaOx-urolithiasis. World J Urol. 2007;25(3):315-323.

17. Siener R, Jahnen A, Hesse A. Influence of a mineral water rich in calcium, magnesium and bicarbonate on urine composition and the risk calcium oxalate crystallization. European Journal of Clinical Nutrition. 2000;58:270-276.

18. Phillips MJ, Cooke JNC. Relation between urinary calcium and sodium in patients with idiopathic hypercalciuria. Lancet. 1967; 24;1(7504):1354-1357.

19. McCarron DA, Rankin LI, Bennett WM, Krutzik S, McClung MR, Luft FC. Urinary calcium excretion at extremes of sodium intake in normal man. Am J Nephrol. 1981;1(2):84-90.

20. Muldowney FP, Freaney R, Moloney MF. Importance of dietary sodium in the hypercalciuria syndrome. Kidney Int. 1982;22(3):292-296.

21. Silver J, Rubinger D, Friedlaender MM, Popovtzer MM. Sodiumdependent idiopathic hypercalciuria in renal-stone formers. Lancet. 1983;2 (8348):484-486.
22. Tratamiento no farmacológico de la hipertensión arterial. Sociedad Española de Hipertensión. http://www.seh-lelha.org/ pdf/guia05_7.pdf

23. Vitoria Miñana I, Arias Jordá T. Importancia Nutricional del agua de consumo público y del agua de bebida envasada en la alimentación del lactante. Estudio descriptivo de base poblacional. IV Premio Especial Nestlé sobre Nutrición Infantil (Asociación Española de Pediatría). http://www.aguainfant. com/BIBLIOGRAF/aguas-2000-i-v.pdf

24. Martin-Gil J, Martin-Ramos P, Martin-Gil FJ. Sobre las aguas minerales naturales de España: asociaciones entre su composición química y localización geográfica. Geographicalia. 1999;37:1-7.

25. Hubert J, Hubert C, Jungers P, Daudon M, Hartemann P. Eaux de boisson et lithiase calcique urinaire idiopathique. Quelles eaux de boisson et quelle cure de diurèse? Progrès en Urologie. 2002;12(4):692-699.

26. Vallejo Herrador J, Galmes Belmonte I, Burgos Revilla FJ, Téllez Martínez-Fornés M, Fernández Fernández E, García Cuerpo E, Escudero Barrilero A. Valor real del aporte de líquidos y sus cualidades, en la población litiásica. Actas Urol Esp. 1992;16(3):233-239.

27. Alapont Pérez FM, Gálvez Calderón J, Varea Herrero J, Colomé Borrós G, Olaso Oltra A, Sánchez Bisono JR. Epidemiología de la litiasis urinaria. Actas Urol Esp. 2001;25(5):341-349.

Correspondencia autor: Dr. Félix Millán Rodríguez

Servicio de Urología. Fundació Puigvert

Cartagena 340-350 - 08025 Barcelona. Tel.: Tel.: 934169700

E-mail autor: fmillan@fundacio-puigvert.es / fmillan@ono.com

Información artículo: Original - Litiasis

Trabajo recibido: marzo 2009

Trabajo aceptado: abril 2009 